\title{
Abdominal adiposity depots are correlates of adverse cardiometabolic risk factors in Caucasian and African-American adults
}

\author{
RL Newton Jr, C Bouchard, G Bray, F Greenway, WD Johnson, E Ravussin, D Ryan \\ and PT Katzmarzyk
}

Pennington Biomedical Research Center, LSU System, Baton Rouge, LA, USA

Objective: Accumulation of adipose tissue is associated with cardiometabolic risks. Although visceral adipose tissue (VAT) has
been strongly implicated in this relationship, there is still some debate regarding the contribution of abdominal subcutaneous
adipose tissue $(S A T)$. The purpose of this study was to determine the contribution of abdominal SAT to cardiometabolic risk
factors, independent of total and visceral adiposity. These relationships were assessed in Caucasian and African Americans.
Design: It is a cross-sectional analysis of the Pennington Center Longitudinal Study.
Subjects: Data were extracted from 1246 participants. Total body fat mass (FM) was measured by dual-energy X-ray absorptio-
metry, whereas abdominal VAT and SAT areas $\left(\mathrm{cm}^{2}\right.$ ) were measured with computed tomography. The cardiometabolic risk
factors included resting blood pressure (BP), fasting blood glucose and triglyceride concentrations and high-density lipoprotein
cholesterol (HDL-C).
Results: Positive relationships across tertiles of VAT were seen for the participants with high glucose, high BP and low HDL-C
$(P<0.043)$. There was also a significant increase in the percentage of participants with two or more cardiometabolic risk factors
across most tertiles of abdominal SAT ( $P<0.042)$. Logistic regression analysis showed that in univariate models, all adiposity
measures were significantly associated with increased odds of having all risk factors in men and women. In multivariate models,
VAT was significantly associated with most risk factors across gender. Abdominal SAT and FM (odds ratios (ORs) $1.3-2.1$; all
$P<0.05)$ were associated with fewer risk factors after accounting for VAT. VAT (OR $=5.9$ and 5.3 ) and SAT (OR $=2.0$ and 1.8$)$
were both associated with higher odds of the presence of two or more cardiometabolic risk factors in both males and females
$(P<0.001)$. Conclusion: The data suggest that abdominal SAT is not protective against unfavorable cardiometabolic risk profiles. These conclusions were consistent across ethnic groups.

Nutrition and Diabetes (2011) 1, e2; doi:10.1038/nutd.2010.2; published online 31 January 2011

Keywords: abdominal fat distribution; heart disease risk; epidemiology; ethnic differences

\section{Introduction}

Abdominal obesity is the hallmark of central or android obesity, and has been suggested as a key component of the metabolic syndrome. ${ }^{1}$ Abdominal fat includes the visceral adipose tissue (VAT) fraction, which has been consistently associated with cardiometabolic risk factors, including

Correspondence: Dr RL Newton Jr, Pennington Biomedical Research Center; Louisiana State University; 6400 Perkins Road; Baton Rouge, LA 70808, USA. E-mail: Robert.Newton@pbrc.edu

The Pennington Center Longitudinal Study is registered at ClinicalTrials.gov (Identifier NCT00959270).

Received 4 August 2010; revised 15 November 2010; accepted 17 November 2010 diabetes, ${ }^{2,3}$ dyslipidemia, ${ }^{4,5}$ atherosclerosis, ${ }^{6}$ decreased insulin sensitivity ${ }^{4,5,7,8}$ and the metabolic syndrome. ${ }^{9,10}$ The relationship between VAT and cardiometabolic risk factors is believed to be because of the fact that VAT produces endocrine, lipolytic and inflammatory factors ${ }^{6,11,12}$ that are known to be associated with insulin resistance and heart disease. It has also been shown that VAT is correlated with ectopic fat deposition in skeletal muscle and liver, a phenomenon that is known to substantially increase the risk of metabolic abnormalities. ${ }^{13}$ These findings have been shown across different age, gender and ethnic groups.

Abdominal subcutaneous adipose tissue (SAT) has also been shown to be positively associated with increased cardiometabolic risk. ${ }^{6,10,12}$ The associations with SAT tend to be weaker than associations between VAT and cardiometabolic 
factors. ${ }^{6,10}$ However, there have been some inconsistent findings regarding the contribution of SAT to the risk of metabolic syndrome and cardiovascular disease. ${ }^{13}$ Gluteal fat has been shown to be protective of cardiometabolic disease. ${ }^{14}$ Some studies have shown few or even no relationships between SAT and the risk factors for cardiometabolic disease. $^{3,7}$ In addition, several epidemiological studies have shown that SAT was negatively associated with risk factors for cardiometabolic disease. ${ }^{5,9,15-17}$ However, these findings were evident only in the highest tertile of VAT, were seen in only one gender group (for example, males) or were seen for only one risk factor (for example, triglycerides). Such findings are consistent with the observation that, in susceptible individuals, excess fat can also be deposited ectopically in the liver, kidney, pancreas, skeletal muscles, heart and so on, ${ }^{11}$ which leads to a decreased ability of these organs to function properly, resulting in a poor cardiometabolic profile. SAT in general, and abdominal SAT in particular, is believed to be the major storage depot for excess fat, preventing ectopic fat deposition. Therefore, one could speculate that a large abdominal SAT for a given level of total adiposity and VAT may be metabolically advantageous and protective.

Given the current uncertainities regarding the contribution of SAT to cardiometabolic risk, the purpose of this study was to assess the relationship between abdominal SAT and cardiometabolic risk factors. Previous studies have conducted similar analyses, but have not controlled for both total and visceral adiposity, confounding the findings. Therefore, we will control for these effects. We also used computed tomography (CT) data to estimate cross-sectional areas of abdominal fat. In addition, our sample is ethnically diverse, which allows for the relationship between adiposity and cardiometabolic risk factors to be assessed across ethnic groups.

\section{Materials and methods}

\section{Study sample}

The Pennington Center Longitudinal Study (PCLS) is designed to assess the effects of lifestyle factors and obesity on the development of chronic disorders such as type 2 diabetes, cardiovascular disease and cancer. The sample is composed of participants who have enrolled in clinical studies at the Pennington Biomedical Research Center (PBRC) since 1992. The current investigation is limited to cross-sectional analyses of individuals who participated in studies requiring a CT scan of the L4-L5 abdominal region, a full-body dual-energy X-ray absorptiometry (DXA) scan, resting blood pressure (BP) and fasting blood draws in which glucose, triglycerides and cholesterol levels were assayed (since 1998). These measurements represented the first observation and, therefore, were obtained before any intervention. There were 1246 adults who met these criteria. Each volunteer provided written informed consent, and all the Pennington Center Longitudinal Study procedures were approved by the Pennington Biomedical Research Center institutional review board.

\section{Measures}

Body composition. Body mass index: Standing height was measured to the nearest $0.1 \mathrm{~cm}$ by a wall-mounted stadiometer (Holtain Ltd, Dyfed, UK). Standard procedures were followed in which the participants removed their shoes, traction was placed on their head in order to align it with the Frankfort Plane and the slide was lowered until it reached the vertex of the skull while the volunteer inhaled. Weight was measured to the nearest $0.1 \mathrm{~kg}$ using a digital scale after heavy outer clothing and pocket items were removed. Two measurements of height and weight were taken. A third measurement was taken if the first two measurements were not within $0.5 \mathrm{~cm}$ or $0.5 \mathrm{~kg}$ of each other. Body mass index was calculated as the weight in kilograms divided by the height in meters squared $\left(\mathrm{kg} \mathrm{m}^{-2}\right)$.

CT: Abdominal VAT and SAT areas $\left(\mathrm{cm}^{2}\right)$ were measured with one of three different CT scanners: GE High Speed Advantage (1998-2000; $n=498)$, GE LightSpeed Plus (2001-2006; $n=697)$, and GE LightSpeed VCT (2007-2008; $n=51$, all from GE Healthcare, Princeton, NJ, USA). Participants lay in a supine position with arms overhead to obtain a cross-sectional image at the L4-L5 intervertebral space. The procedures for defining VAT and SAT have been described by Smith et al. ${ }^{5}$ CT scanners were calibrated daily to air. CT imaging was analyzed with commercially available software (Analyze Direct, Rochester, MN, USA).

$D X A$ : Total body fat mass (FM; $\mathrm{kg}$ ) was measured using a whole-body DXA scanner. FM was calculated as percentage body fat (\%fat) $\times$ scale weight, where \%fat was derived from DXA and the scale weight was the weight measured from a digital scale. One of two Hologic models, QDR 2000 and QDR 4500 (Hologic, Bedford, MA, USA), was used for imaging. The QDR $2000(n=537)$ was used until 2006. The QDR $4500(n=709)$ was used along with the QDR 2000 from 2001 to 2006, and then used exclusively thereafter. A subsample of participants $(n=32)$ had DXA scans conducted by both machines in order to assess comparability. The agreement for \%fat between the machines was high $\left(R^{2}=0.987\right)$, and an equation was developed to convert the QDR 2000 data to be comparable with QDR 4500 data $(Y=0.8015 X+2.3903)$. Phantoms were used for calibration and documentation of the stability of measures over time. Each participant's scan was analyzed with the latest software QDR for Windows V11.2 (Hologic).

Cardiometabolic risk factors. BP: All BP measurements were taken manually using a stethoscope and standard sphygmomanometer, or in some cases using a validated Omron automatic measuring device (Omron, Bannockburn, IL, USA). Resting BP measurements were obtained after a 5-min rest, with the participant in a semi-recumbent position in a quiet room. Each measurement was taken 
twice. The nurse waited for $1-2$ min before repeating the measurement, and the average of the two measurements was recorded. Participants were not allowed to engage in vigorous exercise, ingest food or caffeine or smoke within $30 \mathrm{~min}$ of measurement. Upper-arm length and circumferences of the right arm were measured in order to establish the appropriate cuff size. The Korotkoff sounds were used to establish the first-and fifth phases, and all measurements were recorded in $\mathrm{mm} \mathrm{Hg}$. High BP was defined as systolic BP $\geqslant 130 \mathrm{~mm} \mathrm{Hg}$ or diastolic BP $\geqslant 85 \mathrm{~mm} \mathrm{Hg}$ or self-reported hypertension (13 individuals).

Blood glucose and lipids: Blood was collected following a 12-h fast. Glucose and lipids were analyzed on a Beckman Coulter DXC600 (Beckman Coulter, Brea, CA, USA). High glucose was defined as fasting plasma glucose $\geqslant 100 \mathrm{mg} \mathrm{dl}^{-1}$ or self-reported treatment with a hypoglycemic agent or insulin. High triglycerides were defined as $\geqslant 150 \mathrm{mg} \mathrm{dl}^{-1}$. Low high-density lipoprotein cholesterol (HDL-C) was defined as $\leqslant 40 \mathrm{mg} \mathrm{dl}^{-1}$ for males or $\leqslant 50 \mathrm{mg} \mathrm{dl}^{-1}$ for females.

Covariates. Age, smoking, menopausal status and ethnicity served as covariates. Age was computed from birth and observation dates. Self-reported smoking status was determined from questionnaire responses during initial telephone screening, and the participants were categorized as nonsmokers, current smokers or former smokers. Participants were classified as 'Yes' or 'No' to being a 'current smoker' for the statistical analyses. Menopausal status (premenopausal/ postmenopausal) was determined in females on the basis of their age and responses to questions regarding their reproductive history. Females aged $\geqslant 55$ years or those who indicated that they can no longer have children because of reaching menopause were considered to be postmenopausal. Participants self-classified themselves as African American or Caucasian.

\section{Statistical analysis}

The associations between adiposity and cardiometabolic risk factors (low HDL-C, high BP, high glucose, high triglycerides) were assessed in two ways. In one set of analyses, risk factor values across tertiles of abdominal SAT were assessed within each tertile of VAT. These means were adjusted for age, body mass index and FM. It is important to adjust for both VAT and FM when assessing the independent effect of SAT because there are strong positive interrelationships between the variables. In another set of analyses, hierarchical multiple logistic regression analyses were used to assess the relationships between adiposity variables and cardiometabolic risk factors. The presence or absence of high BP (systolic $\mathrm{BP} \geqslant 130 \mathrm{~mm} \mathrm{Hg}$ or diastolic $\mathrm{BP} \geqslant 85 \mathrm{~mm} \mathrm{Hg}$ ), high triglycerides $\left(\geqslant 150 \mathrm{mg} \mathrm{dl}^{-1}\right)$, low HDL-C $\left(\leqslant 40 \mathrm{mg} \mathrm{dl}^{-1}\right.$ for males and $\leqslant 50 \mathrm{mg} \mathrm{dl}^{-1}$ for females), high low-density lipoprotein (fasting glucose $\geqslant 100 \mathrm{mg} \mathrm{dl}^{-1}$ ) and the presence or absence of two or more cardiometabolic risk factors were modeled as dependent variables. Demographic and adiposity variables served as independent variables. The adiposity measures, SAT, VAT and FM, were modeled as continuous variables. Six models were investigated. Model 1 included the demographic variables age, smoking status, ethnicity and menopausal status. VAT was added to the demographic variables in Model 2 and abdominal SAT was added to the demographic variables in Model 3. In Model 4, both VAT and SAT were entered into the regression analysis along with the demographic variables. Model 5 included the demographic variables and FM and Model 6 included the demographic variables and both FM and VAT.

Correlations were run to determine the association among the adiposity variables. We assessed multicollinearity, which is a measure of how much a given variable is explained by the other independent variables, to determine whether certain independent variables should be excluded from appearing together in the logistic regression models. We considered multicollinarity to be present if tolerance was $<0.10{ }^{18}$ SAS version 9.0 (Cary, NC, USA) was used for all statistical analyses. The level of significance was set at 0.05 .

\section{Results}

A summary of the characteristics of the sample is presented in Table 1 . Approximately $40 \%$ of the sample were male and $33 \%$ were African Americans. The average age was 44.5 years. The overall mean for VAT was $118 \mathrm{~cm}^{2}, 374 \mathrm{~cm}^{2}$ for SAT and $28 \mathrm{~kg}$ of FM.

Age, ethnicity and FM-adjusted means (Table 2), and the percentage of participants (Table 3) with each risk factor, are reported by tertiles of SAT within tertiles of VAT. The only risk factor to decrease significantly across levels of SAT was high BP. This occurred in females within the lowest VAT tertile $(P=0.043)$. Otherwise, the percentages of males with high BP $(P=0.031)$ and females with low HDL-C $(P=0.028)$ increased across levels of SAT in the middle and highest VAT tertiles, respectively. In addition, the percentage of males and females having two or more cardiometabolic risk factors increased significantly across tertiles of SAT in each VAT tertile $(P<0.042)$, except for men in the highest VAT tertile $(P=0.058)$.

SAT and FM were highly correlated $(r=0.93 ; P<0.001)$. The correlations between VAT and SAT $(r=0.36)$ and between VAT and FM $(r=0.53)$ were also significant. Tolerance values for SAT and FM were $<0.083$ in males, and were 0.14 and 0.11 in females, respectively. Although the values in females do not reach the threshold for tolerance $(<0.10)$, greater than $85 \%$ of the variance in SAT and FM is explained by the other independent variables. Therefore, SAT and FM were not included together in the logistic regression models for either gender group.

Table 4 displays the odds ratios (ORs) for the relationship between the adiposity measures and the cardiometabolic risk factors. In Model 1, which included only demographic variables, age was significantly associated with increased 
Table 1 Anthropometric, clinical and biochemical characteristics (means (s.d.)) of the sample stratified by gender and ethnicity

\begin{tabular}{|c|c|c|c|c|c|}
\hline & \multirow[t]{2}{*}{ Overall } & \multicolumn{2}{|c|}{ Males } & \multicolumn{2}{|c|}{ Females } \\
\hline & & African American & Caucasian & African American & Caucasian \\
\hline \multicolumn{6}{|c|}{ Mean (s.d.) } \\
\hline$N$ & 1246 & 100 & 406 & 311 & 429 \\
\hline Age (years) & $44.5(13.1)$ & $36.5(13.8)^{\mathrm{a}}$ & $44.4(13.5)$ & $40.2(11.6)^{a}$ & $49.6(11.5)$ \\
\hline $\mathrm{BMI}\left(\mathrm{kg} \mathrm{m}^{-2}\right)$ & $29.7(5.1)$ & $29.3(5.2)$ & $30.0(4.6)$ & $30.6(5.4)^{\mathrm{a}}$ & $29.0(5.1)$ \\
\hline $\mathrm{SBP}(\mathrm{mm} \mathrm{Hg})$ & $120.5(13.1)$ & $122.1(11.1)$ & $121.6(12.1)$ & $119.0(13.7)$ & $120.0(13.9)$ \\
\hline $\mathrm{DBP}(\mathrm{mm} \mathrm{Hg})$ & $76.6(8.4)$ & $76.8(9.1)$ & $78.0(8.2)$ & $77.2(8.8)^{\mathrm{a}}$ & $74.9(7.8)$ \\
\hline Glucose $\left(\mathrm{mmoll}^{-1}\right)$ & $5.6(1.0)$ & $5.6(0.9)$ & $5.8(1.2)$ & $5.5(0.9)$ & $5.5(0.8)$ \\
\hline Triglycerides $\left(\mathrm{mmoll}^{-1}\right)$ & $1.5(0.9)$ & $1.2(0.8)^{\mathrm{a}}$ & $1.8(1.1)$ & $1.0(0.6)^{a}$ & $1.5(0.9)$ \\
\hline $\mathrm{HDL}-\mathrm{C}\left(\mathrm{mmoll} \mathrm{I}^{-1}\right)$ & $1.4(0.4)$ & $1.2(0.3)$ & $1.2(0.3)$ & $1.5(0.4)$ & $1.5(0.4)$ \\
\hline SAT $\left(\mathrm{cm}^{2}\right)$ & $374.1(153.6)$ & $283.0(163.0)$ & $309.1(136.8)$ & $441.8(147.9)^{a}$ & $407.7(136.0)$ \\
\hline VAT $\left(\mathrm{cm}^{2}\right)$ & $117.8(64.9)$ & $90.3(57.9)^{a}$ & $140.9(70.8)$ & $91.0(49.2)^{\mathrm{a}}$ & $121.8(61.2)$ \\
\hline $\mathrm{FM}(\mathrm{kg})$ & $28.4(9.7)$ & $21.3(9.9)^{a}$ & $25.9(9.2)$ & $31.3(9.4)$ & $30.2(8.9)$ \\
\hline \multicolumn{6}{|c|}{$\%$} \\
\hline High glucose $\mathrm{b}^{\mathrm{b}}$ & 39.6 & 45.0 & 47.8 & 34.1 & 34.5 \\
\hline High $\mathrm{BP}^{\mathrm{C}}$ & 33.5 & 38.0 & 34.5 & 30.9 & 33.3 \\
\hline Smoking $^{d}$ & 4.6 & $12.0^{\mathrm{a}}$ & 2.2 & 4.8 & 4.9 \\
\hline Post menopause & 33.8 & - & - & $11.6^{\mathrm{a}}$ & 49.9 \\
\hline
\end{tabular}

Abbreviations: BP, blood pressure; BMI, body mass index; DBP, diastolic blood pressure; FM, total body fat mass; HDL-C, high-density lipoprotein cholesterol;

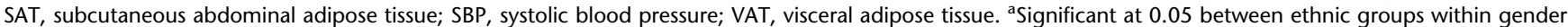
${ }^{\mathrm{b}} \mathrm{High}$ glucose -'Yes' if glucose $\geqslant 100 \mathrm{mg} \mathrm{dl}^{-1}\left(55.5 \mathrm{mmoll}^{-1}\right)$ or self-reported diabetes. 'High BP-'Yes' if SBP $\geqslant 130 \mathrm{~mm} \mathrm{Hg}$ or DBP $\geqslant 85 \mathrm{~mm} \mathrm{Hg}$ or self-reported hypertension. ' Smoking - 'Yes' if current smoker or self-reported during phone interview for recruitment. 'Post menopause-'Yes' if female aged $>54$ years or reported post menopause.

Table 2 Age, ethnicity and FM-adjusted risk factor means by sex-specific SAT tertiles within sex-specific VAT tertiles

\begin{tabular}{|c|c|c|c|c|c|c|c|c|}
\hline & \multicolumn{4}{|c|}{ Males } & \multicolumn{4}{|c|}{ Females } \\
\hline & SAT tertile 1 & SAT tertile 2 & SAT tertile 3 & P-value & SAT tertile 1 & SAT tertile 2 & SAT tertile 3 & $\mathrm{P}$-value \\
\hline \multicolumn{9}{|l|}{ VAT tertile 1 (mean \pm s.e.m.) } \\
\hline $\mathrm{SBP}(\mathrm{mm} \mathrm{Hg})$ & $119.9 \pm 2.1$ & $119.9 \pm 1.5$ & $116.9 \pm 2.3$ & 0.595 & $116.3 \pm 2.0$ & $116.2 \pm 1.4$ & $112.5 \pm 2.1$ & 0.344 \\
\hline $\mathrm{DBP}(\mathrm{mm} \mathrm{Hg})$ & $75.6 \pm 1.5$ & $73.7 \pm 1.1$ & $73.1 \pm 1.7$ & 0.519 & $74.8 \pm 1.4$ & $73.9 \pm 0.9$ & $73.2 \pm 1.4$ & 0.811 \\
\hline Glucose $\left(\mathrm{mmoll}^{-1}\right)$ & $5.3 ; 5.1-5.4$ & $5.4 ; 5.3-5.5$ & $5.3 ; 5.1-5.5$ & 0.251 & $5.0 ; 4.9-5.1$ & $5.1 ; 5.0-5.2$ & $5.2 ; 5.0-5.3$ & 0.400 \\
\hline Triglyceride $\left(\mathrm{mmoll}^{-1}\right)$ & $0.78 ; 0.65-0.93$ & $0.94 ; 0.83-1.06$ & $1.08 ; 0.89-1.31$ & 0.114 & $0.75 ; 0.65-0.87$ & $0.78 ; 0.71-0.87$ & $0.94 ; 0.81-1.10$ & 0.127 \\
\hline \multicolumn{9}{|l|}{ VAT tertile 2 (mean \pm s.e.m.) } \\
\hline $\mathrm{SBP}(\mathrm{mm} \mathrm{Hg})$ & $119.3 \pm 2.3$ & $122.6 \pm \pm 1.8$ & $123.8 \pm 2.3$ & 0.384 & $122.0 \pm 1.9$ & $119.0 \pm 1.3$ & $119.1 \pm 1.9$ & 0.411 \\
\hline $\mathrm{DBP}(\mathrm{mm} \mathrm{Hg})$ & $76.4 \pm 1.6$ & $79.9 \pm 1.2$ & $80.6 \pm 1.6$ & 0.123 & $76.9 \pm 1.2$ & $76.6 \pm 0.8$ & $76.0 \pm 1.2$ & 0.911 \\
\hline Glucose $\left(\mathrm{mmoll}^{-1}\right)$ & $5.6 ; 5.3-5.9$ & $5.8 ; 5.5-6.0$ & $5.9 ; 5.6-6.2$ & 0.406 & $5.4 ; 5.2-5.6$ & $5.5 ; 5.3-5.6$ & $5.4 ; 5.2-5.6$ & 0.936 \\
\hline Triglyceride $\left(\mathrm{mmoll}^{-1}\right)$ & $1.20 ; 0.99-1.46$ & $1.42 ; 1.22-1.64$ & $1.49 ; 1.23-1.80$ & 0.291 & $1.19 ; 1.02-1.37$ & $1.20 ; 1.08-1.33$ & $1.00 ; 0.86-1.16$ & 0.145 \\
\hline \multicolumn{9}{|l|}{ VAT tertile 3 (mean \pm s.e.m.) } \\
\hline $\mathrm{SBP}(\mathrm{mm} \mathrm{Hg})$ & $125.2 \pm 2.8$ & $124.0 \pm 2.1$ & $128.1 \pm 2.7$ & 0.369 & $126.5 \pm 2.1$ & $121.9 \pm 1.5$ & $123.1 \pm 2.1$ & 0.160 \\
\hline $\mathrm{DBP}(\mathrm{mm} \mathrm{Hg})$ & $81.6 \pm 1.9$ & $81.6 \pm 1.4$ & $81.6 \pm 1.8$ & 0.999 & $78.4 \pm 1.2$ & $76.4 \pm 0.9$ & $77.5 \pm 1.2$ & 0.295 \\
\hline Glucose $\left(\mathrm{mmoll}^{-1}\right)$ & $6.3 ; 5.7-6.9$ & $5.8 ; 5.4-6.2$ & $6.0 ; 5.5-6.6$ & 0.121 & $6.1 ; 5.8-6.4$ & $6.0 ; 5.7-6.2$ & $5.6 ; 5.4-5.9$ & 0.160 \\
\hline Triglyceride $\left(\mathrm{mmoll}^{-1}\right)$ & $1.76 ; 1.38-2.24$ & $1.81 ; 1.50-2.17$ & $1.85 ; 1.46-2.34$ & 0.961 & $1.36 ; 1.17-1.57$ & $1.35 ; 1.21-1.51$ & $1.53 ; 1.32-1.78$ & 0.418 \\
\hline
\end{tabular}

Abbreviations: DBP, diastolic blood pressure; FM, total body fat mass; SAT, subcutaneous abdominal adipose tissue; SBP, systolic blood pressure; VAT, visceral adipose tissue.

odds of having two or more cardiometabolic risk factors for both males and females $(P<0.001)$. In Model 2, VAT was added to the model with the demographic variables and was significantly associated with increased odds of having each individual risk factor in men $(\mathrm{OR}=1.4-2.6 ; P<0.05)$ and women $(\mathrm{OR}=1.8-2.9 ; P<0.001)$. VAT was also significantly associated with increased odds of having two or more risk factors across both genders (males: $\mathrm{OR}=10.1$; $95 \%$ confidence interval $(\mathrm{CI}), 6.5-15.7$; females: $\mathrm{OR}=8.1$; $95 \% \mathrm{CI}$, 5.8-11.4). SAT was significantly associated with increased odds of having each individual risk factor in men $(\mathrm{OR}=$ $1.4-1.8 ; P<0.05)$ and women $(\mathrm{OR}=1.5-1.7 ; P<0.001)$ in Model 3, in which it was added to the demographic variables. SAT was also associated with increased odds of 
Table 3 Age, ethnicity and FM-adjusted risk factor percentages by sex-specific SAT tertiles within sex-specific VAT tertiles

\begin{tabular}{|c|c|c|c|c|c|c|c|c|}
\hline & \multicolumn{4}{|c|}{ Males } & \multicolumn{4}{|c|}{ Females } \\
\hline & SAT tertile 1 & SAT tertile 2 & SAT tertile 3 & P-value & SAT tertile 1 & SAT tertile 2 & SAT tertile 3 & $\mathrm{P}$-value \\
\hline \multicolumn{9}{|l|}{ VAT tertile 1 (\%) } \\
\hline High $\mathrm{BP}^{\mathrm{a}}$ & 14.0 & 19.6 & 21.4 & 0.581 & 19.5 & 19.3 & 7.4 & 0.043 \\
\hline High glucose $\mathrm{e}^{\mathrm{b}}$ & 12.3 & 33.9 & 33.9 & 0.008 & 7.3 & 12.1 & 12.4 & 0.515 \\
\hline High triglycerides ${ }^{c}$ & 5.3 & 10.7 & 10.7 & 0.499 & 3.7 & 7.2 & 8.6 & 0.449 \\
\hline Low HDL-C ${ }^{d}$ & 10.5 & 17.9 & 25.0 & 0.138 & 11.0 & 20.5 & 22.2 & 0.122 \\
\hline $2+$ Risk factors ${ }^{\mathrm{e}}$ & 7.0 & 25.0 & 30.4 & 0.003 & 6.1 & 15.7 & 32.1 & $<0.001$ \\
\hline \multicolumn{9}{|l|}{ VAT tertile $2(\%)$} \\
\hline High $B P^{a}$ & 22.8 & 33.9 & 46.4 & 0.031 & 30.5 & 33.7 & 35.4 & 0.811 \\
\hline High glucose $\mathrm{e}^{\mathrm{b}}$ & 61.4 & 53.6 & 58.9 & 0.700 & 34.2 & 34.9 & 28.1 & 0.598 \\
\hline High triglycerides ${ }^{c}$ & 31.6 & 46.4 & 39.3 & 0.288 & 23.2 & 28.9 & 20.7 & 0.486 \\
\hline Low HDL-C ${ }^{d}$ & 19.3 & 30.4 & 30.4 & 0.290 & 18.3 & 28.9 & 34.2 & 0.060 \\
\hline $2+$ Risk factors $^{\mathrm{e}}$ & 42.1 & 67.9 & 80.4 & $<0.001$ & 40.2 & 55.4 & 70.7 & $<0.001$ \\
\hline \multicolumn{9}{|l|}{ VAT tertile $3(\%)$} \\
\hline High $\mathrm{BP}^{\mathrm{a}}$ & 48.2 & 51.8 & 58.9 & 0.556 & 51.9 & 42.9 & 50.0 & 0.475 \\
\hline High glucose $\mathrm{e}^{\mathrm{b}}$ & 62.5 & 51.8 & 57.1 & 0.539 & 58.0 & 64.3 & 57.3 & 0.608 \\
\hline High triglycerides ${ }^{c}$ & 66.1 & 60.7 & 55.4 & 0.531 & 48.2 & 41.7 & 45.1 & 0.715 \\
\hline Low HDL-C ${ }^{d}$ & 30.4 & 37.5 & 37.5 & 0.681 & 30.9 & 38.1 & 51.2 & 0.028 \\
\hline $2+$ Risk factors $^{\mathrm{e}}$ & 85.7 & 96.4 & 96.4 & 0.058 & 74.1 & 84.5 & 89.0 & 0.041 \\
\hline
\end{tabular}

Abbreviations: BP, blood pressure; FM, total body fat mass; HDL-C, high-density lipoprotein cholesterol; SAT, subcutaneous abdominal adipose tissue; VAT, visceral

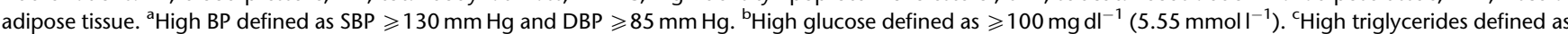

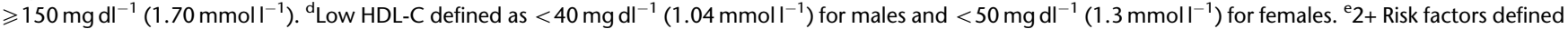
as two or more of high BP, high glucose, high triglycerides or low HDL-C.

Table 4 Odds ratios $(95 \% \mathrm{Cl})$ of having specific cardiometabolic risk factors per standard deviation of VAT, SAT, or FM

\begin{tabular}{|c|c|c|c|c|c|c|}
\hline & \multicolumn{3}{|c|}{ Males } & \multicolumn{3}{|c|}{ Females } \\
\hline & OR $(95 \% \mathrm{Cl})$ & OR $(95 \% \mathrm{Cl})$ & OR $(95 \% \mathrm{Cl})$ & OR $(95 \%$ Cl) & OR $(95 \% \mathrm{Cl})$ & OR $(95 \% \mathrm{Cl})$ \\
\hline$V A T$ & Model 2 & Model 4 & Model 6 & Model 2 & Model 4 & Model 6 \\
\hline High triglyceride & $2.6(2.0-3.3)^{\star *}$ & $2.6(1.9-3.6)^{\star \star}$ & $2.6(1.8-3.7)^{\star \star}$ & $2.3(1.9-2.8)^{\star \star}$ & $2.1(1.7-2.7)^{\star \star}$ & $2.2(1.7-2.9)^{\star *}$ \\
\hline Low HDL & $1.8(1.4-2.2)^{\star \star}$ & $1.5(1.2-2.0)^{\star}$ & $1.5(1.1-2.1)^{*}$ & $2.4(2.0-3.0)^{\star *}$ & $2.2(1.7-2.8)^{\star \star}$ & $2.2(1.7-2.9)^{\star *}$ \\
\hline Diabetes & $1.4(1.1-1.8)^{\star}$ & $1.2(0.9-1.6)$ & $1.1(0.8-1.4)$ & $2.9(2.3-3.7)^{\star \star}$ & $3.0(2.3-3.9)^{\star \star}$ & $2.9(2.2-4.0)^{\star \star}$ \\
\hline High BP & $2.2(1.7-2.8)^{* *}$ & $1.8(1.3-2.4)^{\star *}$ & $1.7(1.2-2.3)^{*}$ & $1.8(1.5-2.2)^{\star *}$ & $1.7(1.4-2.2)^{\star * *}$ & $1.6(1.2-2.1)^{*}$ \\
\hline $2+$ Risk factors & $10.1(6.5-15.8)^{\star \star}$ & $5.9(3.7-9.7)^{\star *}$ & $5.3(3.1-9.1)^{\star \star}$ & $8.1(5.8-11.4)^{\star \star}$ & $5.3(3.6-7.7)^{\star *}$ & $4.3(2.9-6.3)^{\star *}$ \\
\hline SAT & Model 3 & Model 4 & & Model 3 & Model 4 & \\
\hline High triglycerides & $1.5(1.2-1.8)^{\star *}$ & $1.0(0.8-1.2)$ & - & $1.7(1.4-2.0)^{\star *}$ & $1.1(0.9-1.4)$ & - \\
\hline Low HDL & $1.5(1.2-1.8)^{* *}$ & $1.2(1.0-1.6)$ & - & $1.7(1.4-2.0)^{\star *}$ & $1.2(0.9-1.4)$ & - \\
\hline Diabetes & $1.4(1.2-1.7)^{*}$ & $1.3(1.0-1.6)^{*}$ & - & $1.7(1.4-2.0)^{\star *}$ & $1.0(0.8-1.2)$ & - \\
\hline High BP & $1.8(1.5-2.2)^{\star *}$ & $1.4(1.1-1.8)^{\star}$ & - & $1.5(1.2-1.8)^{\star \star}$ & $1.1(0.9-1.4)$ & - \\
\hline $2+$ Risk factors & $4.0(2.9-5.3)^{\star *}$ & $2.0(1.4-2.7)^{\star *}$ & & $3.3(2.7-4.1)^{\star *}$ & $1.8(1.4-2.3)^{\star *}$ & - \\
\hline$F M$ & Model 5 & & Model 6 & Model 5 & & Model 6 \\
\hline High triglycerides & $1.7(1.4-2.1)^{\star *}$ & - & $1.0(0.7-1.3)$ & $1.7(1.4-2.1)^{\star *}$ & - & $1.1(0.8-1.4)$ \\
\hline Low HDL & $1.5(1.3-1.9)^{\star \star}$ & - & $1.2(0.9-1.6)$ & $1.7(1.5-2.1)^{\star *}$ & - & $1.1(0.9-1.4)$ \\
\hline Diabetes & $1.5(1.2-1.9)^{\star *}$ & - & $1.5(1.1-1.9)^{\star}$ & $1.9(1.5-2.2)^{\star \star}$ & - & $1.0(0.8-1.3)$ \\
\hline High BP & $1.9(1.5-2.3)^{\star *}$ & - & $1.4(1.0-1.8)^{*}$ & $1.6(1.3-1.9)^{\star *}$ & - & $1.2(0.9-1.5)$ \\
\hline $2+$ Risk factors & $4.6(3.4-6.2)^{\star *}$ & & $2.0(1.4-2.9)^{\star \star}$ & $4.2(3.3-5.3)^{\star *}$ & & $2.1(1.6-2.8)^{\star *}$ \\
\hline
\end{tabular}

Abbreviations: $\mathrm{BP}$, blood pressure; $\mathrm{Cl}$, confidence interval; FM, total body fat mass; HDL, high-density lipoprotein; OR, odds ratio; SAT, subcutaneous abdominal adipose tissue; VAT, visceral adipose tissue. Odds ratios of having specific risk factor per standard deviation of VAT, SAT or FM unadjusted for other adiposity variables in (A) Model 2 (demographic variables + VAT), Model 3 (demographic variables + SAT) and Model 5 (demographic variables + FM), and adjusted for other adiposity variables in (B) Model 4 (demographic variables + VAT and SAT) and (C) Model 6 (demographic variables + VAT and FM). High triglycerides, triglycerides $\geqslant 150 \mathrm{mg} \mathrm{dl}^{-1}$; Low HDL, HDL $<50 \mathrm{mg} \mathrm{dl}^{-1}$ (women), HDL $<40 \mathrm{mg} \mathrm{dl}^{-1}$ (men); Diabetes, glucose $\geqslant 126 \mathrm{mg} \mathrm{dl}^{-1}$ or reported diabetes; High BP, systolic BP $\geqslant 140 \mathrm{~mm} \mathrm{Hg}$ or diastolic $\mathrm{BP} \geqslant 90 \mathrm{~mm}$ Hg or reported hypertension. 2+ Risk factors, having two or more of high triglyceride, low HDL, diabetes or high $B P$. ${ }^{\star} P<0.05$; $* * P<0.0001$. 
having two or more cardiometabolic risk factors in males $(\mathrm{OR}=4.0 ; 95 \% \mathrm{CI}, 2.9-5.3)$ and females $(\mathrm{OR}=3.3 ; 95 \% \mathrm{CI}$, 2.7-4.1). When both VAT and SAT were included in the same model (Model 4), VAT $(\mathrm{OR}=1.5-2.6 ; P<0.05)$ was significantly associated with increased odds of having high triglycerides, low HDL-C and high $\mathrm{BP}$, and SAT $(\mathrm{OR}=1.3 ; 1.4$; $P<0.05)$ was significantly associated with diabetes and high $\mathrm{BP}$ in men. In women, VAT $(\mathrm{ORs}=1.7-3.0 ; P<0.001)$ was significantly associated with all risk factors, whereas SAT was not associated with any risk factor. Both VAT (males: $\mathrm{OR}=6.0$; 95\% CI, 3.7-9.7; females: $\mathrm{OR}=5.3$; 95\% CI, 3.6-7.6) and SAT (males: $\mathrm{OR}=2.0$; 95\% CI, 1.4-2.7; females: $\mathrm{OR}=1.8 ; 95 \% \mathrm{CI}, 1.4-2.3)$ were significantly associated with having two or more risk factors $(P<0.001)$.

In Model 5, FM was added to the demographic variables and was significantly associated with all individual risk factors in men $(\mathrm{OR}=1.5-1.9 ; \quad P<0.001)$ and women $(\mathrm{OR}=1.6-1.9 ; P<0.001)$. FM was also significantly associated with the presence of two or more risk factors (males: $\mathrm{OR}=4.7 ;$ 95\% CI, 3.4-6.4; females: $\mathrm{OR}=4.3 ; 95 \%$ CI, 3.4-5.4; $P<0.001)$. In Model 6, in which both VAT and FM were assessed simultaneously, VAT was significantly associated with all risk factors in men (except diabetes; $\mathrm{OR}=1.5-2.6 ; P<0.05)$ and women $(\mathrm{OR}=1.6-2.9 ; P<0.05)$. FM was significantly associated with diabetes and high BP in men $(\mathrm{OR}=1.4 ; 1.5 ; P<0.05)$, but was not associated with any risk factor in women. Furthermore, both VAT (males: $\mathrm{OR}=5.2$; 95\% CI, 3.0-9.1; females: $\mathrm{OR}=4.3$; 95\% CI, 2.9-6.3) and FM (males: $\mathrm{OR}=2.0$; 95\% CI, 1.4-3.0; females: $\mathrm{OR}=2.2$; 95\% CI, 1.6-2.9) were significantly associated with increased odds of having two or more risk factors $(P<0.001)$.

In all models, age and ethnicity were significantly associated with increased odds of having one or more cardiometabolic risk factors (data not shown). Given these relationships, we conducted the logistic regression analyses separately for all significant age and ethnicity effects. There were three significant age by VAT interaction effects: high BP in men $(P=0.037)$, high triglycerides in women $(P=0.004)$ and low HDL in women $(P=0.036)$.

\section{Discussion}

Our results showed that abdominal SAT is positively associated with increased odds of having two or more cardiometabolic risk factors. The findings from two different analyses lead to this conclusion. The logistic regression showed that SAT was associated with increased odds favoring the presence of two or more cardiometabolic risk factors. Specifically, every standard deviation increase of SAT was associated with double the odds of having two or more cardiometabolic risk factors. The tertile analysis descriptively showed that the percentage of individuals having two or more cardiometabolic risk factors increased across SAT and VAT tertiles. These effects were shown in both males and females.
There was little evidence in this study that abdominal SAT was associated with a reduced cardiometabolic risk profile, which has been proposed by others. ${ }^{5,9,15-17}$ Of the four risk factors assessed, only the percentage of females with high BP decreased significantly across levels of SAT, and this finding was only shown within the lowest VAT tertile. The logistic regression analyses showed that SAT was associated with increased odds of having each individual risk factor in univariate analyses and increased risk of having two or more risk factors whether or nor VAT was accounted for. Other studies assessing the relationship between abdominal SAT and cardiometabolic risk factors have also shown few protective effects of abdominal SAT. Porter et al. ${ }^{17}$ showed increased odds of meeting criteria for the metabolic syndrome across the middle and highest tertiles of SAT. In addition, a reduced cardiometabolic risk profile across levels of SAT has been demonstrated only at the highest VAT tertiles, ${ }^{17,19}$ only in males ${ }^{17,19}$ or has only been shown with select outcomes such as lower triglycerides. ${ }^{17,20}$ Beyond these specific findings, the vast majority of results in previous studies showed either a non-significant or a positive association between abdominal SAT and cardiometabolic risk factors. Therefore, our results are strongly supportive of the view that abdominal SAT worsens the cardiometabolic risk profile.

One goal of the study was to assess the effect of ethnicity on the odds of having one or more cardiometabolic risk factors. The initial set of regression analyses showed that ethnicity was significantly associated with increased odds of having one or more metabolic risk factors in males and females. These associations were not surprising because African Americans have a higher prevalence of hypertension, diabetes and low-density lipoprotein and lower levels of triglycerides. However, the vast majority of subsequent regression analyses, including the interaction terms for ethnicity, showed few significant effects. These findings show that the observed relationships do not differ significantly by ethnicity.

Our study has several strengths. There were a large number of African-American participants in the cohort, which allowed us to take ethnicity into account when conducting the analyses. In addition, our study employed imaging techniques to assess adiposity. Finally, our tertile analysis adjusted for FM, which has seldom been reported. ${ }^{17}$ Controlling for FM is important to understand the independent effect of SAT, as FM increased across tertiles of SAT within tertiles of VAT (data not shown).

The study was mainly limited by the non-randomly selected sample. The participants in the cohort were volunteers for studies conducted at the Pennington Biomedical Research Center and their characteristics are unlikely to be representative of those of the Louisiana or US population, which limits the generalization of our findings. There was some within-instrument variation across the study time period. For example, there were two different DXA scanners, three CT scanners and two different BP monitoring 
devices. This could potentially introduce bias, although all measurement instruments were calibrated against one another. Additionally, although state-of-the-art imaging measurements were conducted, we did not have a quantification of the deep and the superficial layers of SAT or assessments for upper trunk and limb adiposity. It has been shown that distinct sub-compartments of abdominal SAT relate differently to disease risk, ${ }^{5,8,21}$ which is an area for further research. Several studies have shown that peripheral SAT is protective of cardiovascular disease risk., ${ }^{7,22}$ The effect for peripheral SAT could very well be different form that of abdominal SAT, ${ }^{23}$ as indicated by the results of this study.

Our study showed that abdominal SAT is adversely associated with cardiometabolic risk factors. Overall, there were unfavorable relationships between abdominal SAT and cardiometabolic risk factors in both males and females, and across both ethnic groups. Higher levels of abdominal SAT was associated with increased odds of having two or more cardiometabolic risk factors. Furthermore, Caucasian females had increased odds with each standard deviation increase in VAT for given levels of FM or abdominal VAT. In sum, this investigation indicates that larger quantities of abdominal SAT contribute to an adverse cardiometabolic profile.

\section{Conflict of interest}

The authors declare no conflicts of interest.

\section{Acknowledgements}

We gratefully acknowledge the contributions of Emily Mire and Connie Murla for data management and the many clinical scientists and staff of the Pennington Biomedical Research Center who have contributed data to the Pennington Center Longitudinal Study, particularly Dr William Cefalu, Dr Nikhil Dhurandhar, Dr Lilian Levitan, Dr Timothy Church, Dr Paula Geiselman, Dr Alok Gupta, Dr Corby Martin, Dr Steve Smith and Dr Thomas Gettys. We also acknowledge Julia St Amant for her expert supervision of the CT acquisition and for the analysis of the majority of the CT scans reported herein. This research was supported by the Pennington Biomedical Research Center. PTK is supported, in part, by the Louisiana Public Facilities Authority Endowed Chair in Nutrition and CB is funded, in part, by George A Bray Jr, Chair in Nutrition. ER is funded, in part, by the Douglas L Gordon Chair in Diabetes and Metabolism.

\section{References}

1 Bruce KD, Byrne CD. The metabolic syndrome: common origins of a multifactorial disorder. Postgrad Med J 2009; 85: 614-621.
2 Bray GA, Jablonski KA, Fujimoto WY, Barrett-Connor E, Haffner S, Hanson RL et al. Relation of central adiposity and body mass index to the development of diabetes in the Diabetes Prevention Program. Am J Clin Nutr 2008; 87: 1212-1218.

3 Anjana M, Sandeep S, Deepa R, Vimaleswaran KS, Farooq S, Mohan V. Visceral and central abdominal fat and anthropometry in relation to diabetes in Asian Indians. Diabetes Care 2004; 27: 2948-2953.

4 Di IN, Mittelman SD, Gilsanz V. Differential effect of marrow adiposity and visceral and subcutaneous fat on cardiovascular risk in young, healthy adults. Int J Obes (Lond) 2008; 32: 1854-1860.

5 Smith SR, Lovejoy JC, Greenway F, Ryan D, deJonge L, de la Bretonne $\mathrm{J}$ et al. Contributions of total body fat, abdominal subcutaneous adipose tissue compartments, and visceral adipose tissue to the metabolic complications of obesity. Metabolism 2001; 50: 425-435.

6 Fantuzzi G, Mazzone T. Adipose tissue and atherosclerosis: exploring the connection. Arterioscler Thromb Vasc Biol 2007; 27: 996-1003.

7 Piche ME, Lapointe A, Weisnagel SJ, Corneau L, Nadeau A, Bergeron $\mathrm{J}$ et al. Regional body fat distribution and metabolic profile in postmenopausal women. Metabolism 2008; 57: 1101-1107.

8 Kelley DE, Thaete FL, Troost F, Huwe T, Goodpaster BH. Subdivisions of subcutaneous abdominal adipose tissue and insulin resistance. Am J Physiol Endocrinol Metab 2000; 278: E941-E948.

9 Goodpaster BH, Krishnaswami S, Harris TB, Katsiaras A, Kritchevsky SB, Simonsick EM et al. Obesity, regional body fat distribution, and the metabolic syndrome in older men and women. Arch Intern Med 2005; 165: 777-783.

10 Fox CS, Massaro JM, Hoffmann U, Pou KM, Maurovich-Horvat P, Liu CY et al. Abdominal visceral and subcutaneous adipose tissue compartments: association with metabolic risk factors in the Framingham Heart Study. Circulation 2007; 116: 39-48.

11 Despres JP, Lemieux I. Abdominal obesity and metabolic syndrome. Nature 2006; 444: 881-887.

12 Ibrahim MM. Subcutaneous and visceral adipose tissue: structural and functional differences. Obes Rev 2009; 11: 11-18.

13 Rasouli N, Molavi B, Elbein SC, Kern PA. Ectopic fat accumulation and metabolic syndrome. Diabetes Obes Metab 2007; 9: 1-10.

14 Mason C, Craig CL, Katzmarzyk PT. Influence of central and extremity circumferences on all-cause mortality in men and women. Obesity (Silver Spring) 2008; 16: 2690-2695.

15 Fujimoto WY, Bergstrom RW, Boyko EJ, Chen KW, Leonetti DL, Newell-Morris L et al. Visceral adiposity and incident coronary heart disease in Japanese-American men. The 10-year follow-up results of the Seattle Japanese-American Community Diabetes Study. Diabetes Care 1999; 22: 1808-1812.

16 Narumi H, Yoshida K, Hashimoto N, Umehara I, Funabashi N, Yoshida $\mathrm{S}$ et al. Increased subcutaneous fat accumulation has a protective role against subclinical atherosclerosis in asymptomatic subjects undergoing general health screening. Int J Cardiol 2009; 135: 150-155.

17 Porter SA, Massaro JM, Hoffmann U, Vasan RS, O'Donnel CJ, Fox CS. Abdominal subcutaneous adipose tissue: a protective fat depot? Diabetes Care 2009; 32: 1068-1075.

18 Lin FJ. Solving multicollinearity in the process of fitting regression model using the nested estimate procedure. Qual Quant 2008; 42: 417-426.

19 Demerath EW, Reed D, Rogers N, Sun SS, Lee M, Choh AC et al. Visceral adiposity and its anatomical distribution as predictors of the metabolic syndrome and cardiometabolic risk factor levels. Am J Clin Nutr 2008; 88: 1263-1271.

20 Oka R, Miura K, Sakurai M, Nakamura K, Yagi K, Miyamoto S et al. Impacts of visceral adipose tissue and subcutaneous adipose tissue on metabolic risk factors in middle-aged Japanese. Obesity (Silver Spring) 2010; 18: 153-160. 
21 Lovejoy JC, Smith SR, Rood JC. Comparison of regional fat distribution and health risk factors in middle-aged white and African American women: The Healthy Transitions Study. Obes Res 2001; 9: 10-16.

22 Snijder MB, Visser M, Dekker JM, Goodpaster BH, Harris TB, Kritchevsky SB et al. Low subcutaneous thigh fat is a risk factor for unfavourable glucose and lipid levels, independently of high abdominal fat. The Health ABC Study. Diabetologia 2005; 48: 301-308.

23 Piernas C, Hernandez-Morante JJ, Canteras M, Zamora S, Garaulet M. New computed tomography-derived indices to predict cardiovascular and insulin-resistance risks in overweight/obese patients. Eur J Clin Nutr 2009; 63: 887-897.

(c)

This work is licensed under the Creative Commons Attribution-NonCommercial-No Derivative Works 3.0 Unported License. To view a copy of this license, visit http://creativecommons.org/ licenses/by-nc-nd/3.0/ 